ANNALES

POLONICI MATHEMATICI

$83.2(2004)$

\title{
Pinceaux de courbes planes et invariants polaires
}

\author{
par Evelia R. García Barroso (Tenerife) \\ et Arkadiusz PŁoski (Kielce)
}

\begin{abstract}
We study pencils of plane curves $f_{t}=f-t l^{N}, t \in \mathbb{C}$, using the notion of polar invariant of the plane curve $f=0$ with respect to a smooth curve $l=0$. More precisely we compute the jacobian Newton polygon of the generic fiber $f_{t}, t \in \mathbb{C}$. The main result gives the description of pencils which have an irreducible fiber. Furthermore we prove some applications of the local properties of pencils to singularities at infinity of polynomials in two complex variables.
\end{abstract}

1. Introduction. Nous appellerons courbe (plane) une série convergente $f \in \mathbb{C}\{x, y\}, f \neq 0$, sans terme constant. Pour toutes séries $f, g \in$ $\mathbb{C}\{x, y\}$ nous notons $(f, g)_{0}=\operatorname{dim}_{\mathbb{C}} \mathbb{C}\{x, y\} /(f, g)$ la multiplicité d'intersection de $f$ avec $g$. Si $f=0$ est une courbe alors $\mu(f)=(\partial f / \partial x, \partial f / \partial y)_{0}$ est son nombre de Milnor. On a $\mu(f)<\infty$ si et seulement si la série $f$ n'a pas de facteurs multiples. Nous dirons alors que la courbe $f=0$ est réduite. Soient $l=0$ une courbe régulière, c'est-à-dire telle que $d l(0,0)=$ $\left(\frac{\partial l}{\partial x}(0,0), \frac{\partial l}{\partial y}(0,0)\right) \neq(0,0)$, et $N \geq 1$ un entier. Nous allons étudier les pinceaux de courbes planes de la forme $\left(f_{t}:=f-t l^{N}: t \in \mathbb{C}\right)$ où $f=0$ est une courbe réduite. De tels pinceaux apparaissent dans l'étude des singularités à l'infini d'un polynôme de deux variables de degré $N$ (voir [Eph], $[\mathrm{LW}],[\mathrm{MM}]$ et la section 6 de cette note). Soit $U \subset \mathbb{C}$ un ouvert de Zariski. Nous dirons que le pinceau $\left(f_{t}: t \in U\right)$ où $f_{t}=f-t l^{N}$ est équisingulier si le nombre de Milnor $\mu\left(f_{t}\right)$ est constant pour $t \in U$. Ceci veut dire grâce au théorème $\mu$-constant (voir $[\mathrm{LR}],[\mathrm{Ca}]$ ) que pour tout $t_{1}, t_{2} \in U$ les courbes $f_{t_{1}}=0$ et $f_{t_{2}}=0$ sont équisingulières au sens de Zariski. Il existe toujours l'ouvert le plus grand avec cette proprieté ([LW]), on l'appelle ouvert d'équisingularité du pinceau $f_{t}=f-t l^{N}$. Son complément s'appelle ensemble des valeurs spéciales. Nous dirons que la fibre $f_{t}$ du pinceau est spéciale si $t$ est une valeur spéciale. En cas contraire nous dirons que $f_{t}$ est une fibre générique du pinceau.

2000 Mathematics Subject Classification: Primary 32S55; Secondary 14H20.

Key words and phrases: pencil of plane curves, polar invariant, equisingularity. 
Ephraim [Eph] a montré qu'un pinceau $f_{t}=f-t l^{N_{0}}, N_{0}=(f, l)_{0}$, dont toutes les fibres sont irréductibles est équisingulier et a appliqué ce résultat aux courbes planes ayant une branche à l'infini. Dans son article il a utilisé la notion d'invariant polaire introduite et étudié dans le cas de singularités isolées d'hypersurfaces par Teissier [T1] et a indiqué que les formules de Merle [Me] (voir aussi [S]) pour les invariants polaires d'une branche plane se généralisent au cas où les courbes $f=0$ et $l=0$ ne sont pas transverses. Supposons que $f=f(x, y)$ est une série réduite et que le jacobien

$$
j(f, l)=\frac{\partial f}{\partial x} \frac{\partial l}{\partial y}-\frac{\partial f}{\partial y} \frac{\partial l}{\partial x}
$$

est sans terme constant. Les nombres rationnels $(f, p)_{0} /(l, p)_{0}$ où $p$ parcourt les facteurs irréductibles de $j(f, l)$ s'appellent invariants polaires de la courbe $f=0$ par rapport à la courbe lisse $l=0$. On a

$$
(f, p)_{0}=\min \left\{\left(\frac{\partial f}{\partial x}, p\right)_{0},\left(\frac{\partial f}{\partial y}, p\right)_{0}\right\}+(l, p)_{0}
$$

et donc tous les invariants polaires d'une courbe singulière $f=0$ sont plus grands que 1 . Il est bien connu que l'ensemble des invariants polaires ne dépend que de la classe d'équisingularité de la courbe $f l=0$ (voir [T1], [LMW], [Ga]). La proposition suivante est due à Ephraim ([Eph, Remark 2.3, p. 355]) qui considérait le cas $N=N_{0}$. Pour le cas général voir [MM] et la section 2 de cette note.

Notons $Q(f, l)$ l'ensemble des invariants polaires d'une courbe réduite (pas nécessairement irréductible) $f=0$ par rapport à la courbe lisse $l=0$.

Proposition 1.1. Considérons une courbe réduite $f=0$ et une branche lisse $l=0$ qui n'est pas une composante de $f=0$. Soit $N>0$ un entier. Alors :

(1) Le pinceau $\left(f-t l^{N}: t \neq 0\right)$ est équisingulier si et seulement si $N \notin Q(f, l)$.

(2) Le nombre 0 est une valeur régulière de $\left(f-t l^{N}: t \in \mathbb{C}\right)$ si et seulement si $\max Q(f, l) \leq N$.

(3) Le pinceau $\left(f-t l^{N}: t \in \mathbb{C}\right)$ est équisingulier si et seulement si $\max Q(f, l)<N$.

Les propriétés (2) et (3) se trouvent déjà dans [T1] pour les singularités isolées d'hypersurfaces.

Notons $\eta(f, l)=\max Q(f, l)$ et $\eta^{\min }=\min _{t \in \mathbb{C}}\left\{\eta\left(f_{t}, l\right)\right\}$. Comme corollaire de la proposition ci-dessus nous avons :

Corollaire 1.2. L'entier $[\eta(f, l)]+1$ est égal au plus petit entier $N>0$ tel que le pinceau $\left(f-t l^{N}: t \in \mathbb{C}\right)$ soit équisingulier. 
Écrivons $j(f, l)=\prod_{i=1}^{s} p_{i}$ avec $p_{i} \in \mathbb{C}\{x, y\}$ irréductible et pour tout $q \in Q(f, l)$ posons

$$
A_{q}:=\left\{i: \frac{\left(f, p_{i}\right)_{0}}{\left(l, p_{i}\right)_{0}}=q\right\}, \quad j_{q}:=\prod_{i \in A_{q}} p_{i} \quad m_{q}:=\left(l, j_{q}\right)_{0} .
$$

Nous appelons $m_{q}$ multiplicité de l'invariant polaire $q$.

Le lemme suivant est dû à Teissier ([T1, remarque 1.4]):

Lemme 1.3. Avec les notations ci-dessus on a:

(1) $\sum_{q \in Q(f, l)} m_{q}=(f, l)_{0}-1$,

(2) $\sum_{q \in Q(f, l)} m_{q}(q-1)=\mu(f)$.

Rappelons maintenant que le polygone de Newton d'une série $g(u, v)=$ $\sum_{i j} a_{i j} u^{i} v^{j} \in \mathbb{C}\{u, v\}$ est défini comme le bord de l'enveloppe convexe dans $\mathbb{R}^{2}$ de l'ensemble $\left\{(i, j): a_{i j} \neq 0\right\}+\mathbb{N}^{2}$. En éliminant les variables $x, y$ des équations $f(x, y)=v, l(x, y)=u, \partial(f, l) / \partial(x, y)=0$ nous obtenons l'équation $\Delta(u, v)=0$ de la courbe discriminante de $f$ par rapport à $l$; son polygone de Newton dans les coordonnées $(u, v)$ est appelé polygone de Newton jacobien $\mathcal{N}(f, l)$ (voir [T1], [T3]). Plus précisément le polygone $\mathcal{N}(f, l)$ est déterminé par les conditions suivantes ([T3, pp. 195-197]) :

(1) les pentes des côtés du polygone $\mathcal{N}(f, l)$ sont égales aux $-1 / q$ où $q \in Q(f, l)$

(2) la longueur de la projection du côté de pente $-1 / q$ sur l'axe vertical est égale à la multiplicité $m_{q}$ du quotient $q$ (et alors la longueur de sa projection sur l'axe horizontal est égale à $\left.m_{q} q=\left(f, j_{q}\right)_{0}\right)$.

D'après le lemme 1.3 le polygone $\mathcal{N}(f, l)$ joint les points $\left(0, \sum_{q \in Q(f, l)} m_{q}\right)$ $=\left(0,(f, l)_{0}-1\right)$ et $\left(\sum_{q \in Q(f, l)} m_{q} q, 0\right)=\left(\mu(f)+(f, l)_{0}-1,0\right)$.

Rappelons que toutes les pentes du polygone $\mathcal{N}(f, l)$ d'une courbe singulière $f=0$ sont plus grandes que -1 . Soit $N>0$ un entier. Notons $\mathcal{N}(f, l)_{N}$ le polygone obtenu à partir du polygone $\mathcal{N}(f, l)$ en remplaçant tous les côtés de pente plus grande ou égale à $-1 / N$ par un côté de pente $-1 / N$. Par conséquent le polygone $\mathcal{N}(f, l)_{N}$ joint les points $\left(0, \sum_{q \in Q(f, l)} m_{q}\right)$ et $\left(\sum_{q \in Q(f, l)} m_{q} \min (q, N), 0\right)$ et il est au-dessous du polygone $\mathcal{N}(f, l)$.

Proposition 1.4. Soit $\mathcal{F}$ le pinceau $\left(f-t l^{N}: t \in \mathbb{C}\right)$ et fixons $t_{0} \in \mathbb{C}$. On $a$ :

(1) Si $t_{0}$ est une valeur régulière du pinceau $\mathcal{F}$ alors $\mathcal{N}\left(f_{t_{0}}, l\right)=\mathcal{N}(f, l)_{N}$.

(2) Si $t_{0}$ est une valeur spéciale du pinceau $\mathcal{F}$ telle que $f_{t_{0}}$ est une fibre réduite alors le polygone $\mathcal{N}\left(f_{t_{0}}, l\right)$ est strictement au-dessus du polygone $\mathcal{N}(f, l)_{N}$. 
La démonstration de la proposition ci-dessus est donnée dans la section 2. En employant le lemme 1.3 nous obtenons la conséquence suivante de la proposition 1.4 :

COROLlaire 1.5. Le nombre de Milnor $\mu^{\text {min }}$ et l'invariant polaire maximal $\eta^{\text {min }}$ d'une fibre générique du pinceau $\mathcal{F}$ sont donnés par les formules suivantes :

(1) $\mu^{\min }=\sum_{q \in Q(f, l)}(\min (q, N)-1) m_{q}$,

(2) $\eta^{\min }=\min (\eta(f, l), N)$.

Si $t$ est une valeur spéciale de $\mathcal{F}$ alors $\mu\left(f_{t}\right)>\mu^{\min }$ et $\eta\left(f_{t}\right)>\eta^{\min }$.

La deuxième partie du corollaire ci-dessus se trouve déjà dans [MM, proposition 1]. D'autre part la proposition 1.4 détermine le polygone de Newton jacobien de toute fibre réduite de $\mathcal{F}$, complétant le résultat de la proposition 2 et le corollaire 2 de $[\mathrm{MM}]$ où ne sont déterminées que les pentes de ce polygone.

La notion de multiplicité de l'invariant polaire permet d'estimer le nombre de valeurs spéciales d'un pinceau.

Proposition 1.6. Supposons que l'entier $N$ est un invariant polaire de la courbe $f=0$ par rapport à la branche lisse $l=0$, de multiplicité $m_{N}$. Alors le pinceau $\left(f-t l^{N}: t \in \mathbb{C}\right)$ a au plus $m_{N}$ valeurs spéciales différentes de zéro.

La démonstration est donnée dans la section 2. Comme corollaire nous obtenons le résultat suivant, bien connu (voir [LVTO] et $[\mathrm{CaP}]$ ) :

Corollaire 1.7. Le pinceau $\left(f-t l^{N}: t \in \mathbb{C}\right)$ possède au plus $(f, l)_{0}-1$ valeurs spéciales.

Présentons maintenant le résultat principal de cette note :

THÉORÈme 1.8. Soient $f=0$ une courbe (pas nécessairement réduite), $l=0$ une branche lisse qui n'est pas une composante de $f=0$, et $N>0$ un nombre entier. Supposons qu'une fibre $f_{t_{0}}=f-t_{0} l^{N}$ du pinceau $\mathcal{F}=$ $\left(f_{t}=f-t l^{N}: t \in \mathbb{C}\right)$ est irréductible.

(1) Si $\eta\left(f_{t_{0}}, l\right)<N$ alors le pinceau $\mathcal{F}$ est équisingulier. En particulier toutes les fibres du pinceau sont irréductibles.

(2) Si $\eta\left(f_{t_{0}}, l\right)=N$ alors il existe une seule valeur spéciale $t_{1} d u$ pinceau $\mathcal{F}$; on a $t_{1} \neq t_{0}$.

(3) Si $\eta\left(f_{t_{0}}, l\right)>N$ et $N \notin Q\left(f_{t_{0}}, l\right)$ alors $t_{0}$ est la seule valeur spéciale du pinceau $\mathcal{F}$. 
(4) Si $\eta\left(f_{t_{0}}, l\right)>N$ et $N \in Q\left(f_{t_{0}}, l\right)$ alors $\mathcal{F}$ a exactement deux valeurs spéciales $t_{0}$ et $t_{1} \neq t_{0}$. Toutes les fibres génériques de $\mathcal{F}$ sont décomposables.

La démonstration du théorème ci-dessus est donnée dans la section 4 de cette note. Nous y donnerons aussi une formule explicite pour la valeur spéciale $t_{1}$. Les exemples illustrant le théorème 1.8 se trouvent dans la section 5 .

Finalement notons le corollaire suivant :

Corollaire 1.9. Soit $\mathcal{F}$ le pinceau $\left(f-t l^{N}: t \in \mathbb{C}\right)$.

(1) Si $\mathcal{F}$ a au moins trois valeurs spéciales alors toutes les fibres de $\mathcal{F}$ sont décomposables.

(2) Si toutes les fibres de $\mathcal{F}$ sont irréductibles alors $\mathcal{F}$ a au plus une valeur spéciale.

2. Valeurs spéciales. Reprenons les notations de l'introduction : $f=$ $f(x, y) \in \mathbb{C}\{x, y\}$ est une série réduite, $l=l(x, y) \in \mathbb{C}\{x, y\}$ une série régulière, et $f_{t}=f-t l^{N}$ où $N>0$ est un nombre entier.

Écrivons $j(f, l)=\prod_{i=1}^{s} p_{i}$ avec $p_{i} \in \mathbb{C}\{x, y\}$ irréductible et posons

$$
\begin{aligned}
I(N) & =\left\{i:\left(f, p_{i}\right)_{0}<N\left(l, p_{i}\right)_{0}\right\}, \\
J(N) & =\left\{i:\left(f, p_{i}\right)_{0}>N\left(l, p_{i}\right)_{0}\right\}, \\
K(N) & =\left\{i:\left(f, p_{i}\right)_{0}=N\left(l, p_{i}\right)_{0}\right\} .
\end{aligned}
$$

De plus pour tout $i \in K(N)$ notons $t_{i}$ le nombre unique tel que

$$
\left(f_{t_{i}}, p_{i}\right)_{0}>\min \left\{\left(f, p_{i}\right)_{0}, N\left(l, p_{i}\right)_{0}\right\}=\left(f, p_{i}\right)_{0}=N\left(l, p_{i}\right)_{0} .
$$

Évidemment $t_{i} \neq 0$.

Proposition 2.1. Avec les notations introduites ci-dessus, nous avons

(1) $\min \left\{\mu\left(f_{t}\right): t \neq 0\right\}=\sum_{i \in I(N) \cup K(N)}\left(f, p_{i}\right)_{0}+N \sum_{i \in J(N)}\left(l, p_{i}\right)_{0}-$ $(f, l)_{0}+1$.

(2) Pour tout $t \in \mathbb{C}-\{0\}$ :

$$
\mu\left(f_{t}\right)>\min \left\{\mu\left(f_{t^{\prime}}\right): t^{\prime} \neq 0\right\} \Leftrightarrow t \in\left\{t_{i}\right\}_{i \in K(N)} .
$$

(3) $\mu(f)-\min \left\{\mu\left(f_{t}\right): t \neq 0\right\}=\sum_{i \in J(N)}\left(\left(f, p_{i}\right)_{0}-N\left(l, p_{i}\right)_{0}\right)$.

Démonstration. Fixons un $t \neq 0$. Il est clair que $\mu\left(f_{t}\right)+(f, l)_{0}-1=$ $\mu\left(f_{t}\right)+\left(f_{t}, l\right)_{0}-1$ et en utilisant la formule de Teissier ([T2, Proposition $1.2 \mathrm{du}$ chapitre 2]), nous obtenons $\mu\left(f_{t}\right)+(f, l)_{0}-1=\left(f_{t}, j\left(f_{t}, l\right)\right)_{0}=$ $\left(f_{t}, j(f, l)\right)_{0}$ car $j\left(f_{t}, l\right)=j(f, l)$. Ainsi nous avons 


$$
\begin{aligned}
\mu\left(f_{t}\right)+(f, l)_{0}-1 & =\sum_{i=1}^{s}\left(f_{t}, p_{i}\right)_{0} \\
& =\sum_{i \in I(N)}\left(f_{t}, p_{i}\right)_{0}+\sum_{i \in J(N)}\left(f_{t}, p_{i}\right)_{0}+\sum_{i \in K(N)}\left(f_{t}, p_{i}\right)_{0} \\
& =\sum_{i \in I(N)}\left(f, p_{i}\right)_{0}+\sum_{i \in J(N)} N\left(l, p_{i}\right)_{0}+\sum_{i \in K(N)}\left(f_{t}, p_{i}\right)_{0} \\
& \geq \sum_{i \in I(N)}\left(f, p_{i}\right)_{0}+N \sum_{i \in J(N)}\left(l, p_{i}\right)_{0}+\sum_{i \in K(N)}\left(f, p_{i}\right)_{0}
\end{aligned}
$$

où la dernière inégalité est stricte si et seulement si $t \in\left\{t_{i}: i \in K(N)\right\}$. Ceci montre (1) et (2). Pour vérifier (3) observons qu'après un calcul analogue nous obtenons

$$
\mu(f)+(f, l)_{0}-1=\sum_{i=1}^{s}\left(f, p_{i}\right)_{0}=\sum_{i \in I(N) \cup J(N) \cup K(N)}\left(f, p_{i}\right)_{0}
$$

et donc $\mu(f)-\min \left\{\mu\left(f_{t}\right): t \neq 0\right\}=\sum_{i \in J(N)}\left(\left(f, p_{i}\right)_{0}-N\left(l, p_{i}\right)_{0}\right)$.

Donnons maintenant la démonstration de la proposition 1.1 :

Démonstration de la proposition 1.1. D'après la proposition 2.1(2) nous avons les équivalences

$$
\begin{aligned}
\left(f-t l^{N}: t \neq 0\right) \text { est équisingulier } & \Leftrightarrow \mu\left(f_{t}\right)=\min \left\{\mu\left(f_{t^{\prime}}\right): t^{\prime} \neq 0\right\} \\
& \Leftrightarrow K(N)=\emptyset \Leftrightarrow N \notin Q(f, l) .
\end{aligned}
$$

Maintenant d'après la proposition 2.1(3) nous avons les équivalences

$$
\begin{aligned}
0 \text { est une valeur régulière } & \Leftrightarrow \mu(f)=\min \left\{\mu\left(f_{t}\right): t \neq 0\right\} \\
& \Leftrightarrow J(N)=\emptyset \Leftrightarrow \max Q(f, l) \leq N .
\end{aligned}
$$

La troisième partie de la proposition est une conséquence de (1) et (2).

Soient maintenant $f / h$ une fraction méromorphe (où $f, h$ sont deux séries premières entre elles) et $g=g(x, y)$ une série irréductible telle que $h \not \equiv 0$ $(\bmod g)$. Soit $(x(u), y(u)) \in \mathbb{C}\{u\}^{2},(x(0), y(0))=(0,0)$, une paramétrisation de la branche $g(x, y)=0$. Posons

$$
\left(\frac{f}{h}\right)(g):=\left.\frac{f(x(u), y(u))}{h(x(u), y(u))}\right|_{u=0} \in \mathbb{C} \cup\{\infty\} .
$$

La proposition ci-dessous est due à Maugendre et Michel (voir [MM, Théorème 1]) qui en ont donné une démonstration topologique.

Proposition 2.2 (sur la description des valeurs spéciales). Soit $\mathcal{F}=$ $\left(f_{t}=f-t l^{N}: t \in \mathbb{C}\right)$ le pinceau défini par une courbe $f(x, y)=0$ et une courbe lisse $l(x, y)=0$ qui n'est pas une composante de $f(x, y)=0$. 
Notons $\mu^{\min }:=\inf \left\{\mu\left(f_{t}\right): t \in \mathbb{C}\right\}$. Alors

$$
\left\{t \in \mathbb{C}: \mu\left(f_{t}\right)>\mu^{\min }\right\}=\left\{\left(\frac{f}{l^{N}}\right)(g): g \in \mathcal{B}\right\}
$$

où $\mathcal{B}$ est l'ensemble des branches $g=0$ de la courbe polaire $j(f, l)=0$ telles que $(f, g)_{0} /(l, g)_{0} \geq N$.

Démonstration. En vertu de la proposition 2.1(2),(3) l'ensemble des valeurs spéciales de $\left(f_{t}: t \neq 0\right)$ est égal à $\left\{t_{i}: i \in K(N)\right\}$ et de plus 0 est une valeur spéciale si et seulement s'il existe une branche $p_{i}$ de $j(f, l)=0$ telle que $\left(f, p_{i}\right)_{0}>\left(l^{N}, p_{i}\right)_{0}$.

Considérons la fraction meromorphe $f / l^{N}$. Il suffit d'observer que $\left(f / l^{N}\right)\left(p_{i}\right)=t_{i}$ si $i \in K(N)$ et $\left(f / l^{N}\right)\left(p_{i}\right)=0$ si $i \in J(N)$.

Rappelons la notation de l'introduction : $j(f, l)=\prod_{q \in Q(f, l)} j_{q}$ et $m_{q}=$ $\left(l, j_{q}\right)_{0}$.

Démonstration du lemme 1.3. En employant deux fois la formule de Teissier nous obtenons

$$
(f, l)_{0}-1=(l, j(f, l))_{0}=\sum_{q \in Q(f, l)}\left(l, j_{q}\right)_{0}=\sum_{q \in Q(f, l)} m_{q},
$$

et

$$
\mu(f)+(f, l)_{0}-1=(f, j(f, l))_{0}=\sum_{q \in Q(f, l)}\left(f, j_{q}\right)_{0}=\sum_{q \in Q(f, l)} m_{q} q .
$$

Démonstration de la proposition 1.4. Fixons un $t_{0} \neq 0$ et observons que

$$
\left(f_{t_{0}}, p\right)_{0}=\left(f-t_{0} l^{N}, p\right)_{0} \geq \min \left\{(f, p)_{0}, N(l, p)_{0}\right\}
$$

avec égalité si $t_{0}$ n'est pas une valeur spéciale. Ainsi

$$
\frac{\left(f_{t_{0}}, p_{i}\right)_{0}}{\left(l, p_{i}\right)_{0}}=\left\{\begin{array}{l}
\min \left\{\frac{\left(f, p_{i}\right)_{0}}{\left(l, p_{i}\right)_{0}}, N\right\} \quad \text { si } \frac{\left(f, p_{i}\right)_{0}}{\left(l, p_{i}\right)_{0}} \neq N \\
N \quad \text { si } \frac{\left(f, p_{i}\right)_{0}}{\left(l, p_{i}\right)_{0}}=N \text { et } t_{0} \text { est une valeur régulière }
\end{array}\right.
$$

et $\left(f_{t_{0}}, p_{i}\right)_{0} /\left(l, p_{i}\right)_{0}>N$ si $\left(f, p_{i}\right)_{0} /\left(l, p_{i}\right)_{0}=N$ et $t_{0}$ est une valeur spéciale.

Supposons maintenant que $t_{0}$ est une valeur régulière. Si $t_{0}=0$ alors d'après la proposition 1.1 on a $\max Q(f, l) \leq N$ et nous avons $\mathcal{N}\left(f_{t_{0}}, l\right)=$ $\mathcal{N}(f, l)=\mathcal{N}(f, l)_{N}$. Si $t_{0} \neq 0$ les formules ci-dessus montrent que $Q\left(f_{t_{0}}, l\right)=$ $\{q \in Q(f, l): q<N\} \cup\{N\}$. Pour tout $q^{\prime} \in Q\left(f_{t_{0}}, l\right)$ la multiplicité de $q^{\prime}$ en tant qu'invariant polaire de $f_{t_{0}}=0$ par rapport à $l=0$ est égale à $m_{q}$ si $q^{\prime}=q<N$ et à $\sum_{q \geq N} m_{q}$ si $q^{\prime}=N$. Ceci montre que $\mathcal{N}\left(f_{t_{0}}, l\right)=\mathcal{N}(f, l)_{N}$. Le cas où $t_{0}$ est une valeur spéciale et la fibre $f_{t_{0}}$ est réduite se démontre d'une façon analogue. 
Démonstration de la proposition 1.6. D'après la proposition 2.2 les valeurs spéciales du pinceau $\left(f-t l^{N}: t \in \mathbb{C}\right)$ différentes de zéro sont de la forme $\left(f / l^{N}\right)\left(p_{i}\right)$ où $\left(f, p_{i}\right)_{0} /\left(l, p_{i}\right)_{0}=N$. On déduit le résultat de la définition de la multiplicité $m_{N}$.

Remarque 2.3. Le cas "dégénéré" de Maugendre-Michel [MM] concerne les valeurs de $N$ qui sont inférieures ou égales au plus petit invariant polaire de $f=0$. D'après la proposition 1.4 le polygone jacobien $\mathcal{N}(f, l)_{N}$ d'une fibre régulière a un côté joignant les points $\left((f, l)_{0}-1,0\right)$ et $\left(0, N(f, l)_{0}-N\right)$. On a $\mu^{\mathrm{min}}=(N-1)\left((f, l)_{0}-1\right)$ et $\eta^{\mathrm{min}}=N$. Si $N<\min Q(f, l)$ il y a une seule valeur spéciale $t_{0}=0$. Par contre si $N=\min Q(f, l)$ alors $t_{0}=0$ est une valeur spéciale si et seulement si $Q(f, l) \neq\{N\}$. De plus il existe toujours au moins une valeur spéciale $t_{1} \neq 0$.

3. Développements de Puiseux. Soit $f=f(x, y) \in \mathbb{C}\{x, y\}$ une série irréductible, $y$-régulière d'ordre $n>0$, c'est-à-dire telle que ord $f(0, y)=n$. D'après le théorème de Newton-Puiseux il existe une série $y(t) \in \mathbb{C}\{t\}$ sans terme constant telle que $f\left(t^{n}, y(t)\right)=0$. De plus toute solution de l'équation $f(x, y)$ est de la forme $y(\varepsilon t)$ avec $\varepsilon \in \mathbb{C}$ tel que $\varepsilon^{n}=1$. Il existe une suite unique d'entiers positifs $b_{1}<\cdots<b_{h}$ telle que $\left\{\operatorname{ord}(y(\varepsilon t)-y(t)): \varepsilon^{n}=1\right\}$ $=\left\{b_{1}, \ldots, b_{h}\right\}$. Nous posons $b_{0}=n$ et appelons la suite $\left(b_{0}, \ldots, b_{h}\right)$ la $c a-$ ractéristique de $f$. Soit $e_{k}=\operatorname{pgcd}\left(b_{0}, \ldots, b_{k}\right)$ pour $k \in\{1, \ldots, h\}$. Posons

$$
\bar{b}_{k}=b_{k}+\frac{1}{e_{k-1}} \sum_{i=1}^{k-1}\left(e_{i-1}-e_{i}\right) b_{i} \quad \text { pour } k \in\{0,1, \ldots, h\}
$$

(en particulier $\bar{b}_{0}=b_{0}$ et $\bar{b}_{1}=b_{1}$ ).

La démonstration du théorème ci-dessous est donnée dans [Me] (voir aussi $[\mathrm{Eph}])$.

Théorème De Smith-Merle-Ephraim. Soit $f=f(x, y)$ une série irréductible de caractéristique $\left(b_{0}, \ldots, b_{h}\right)$ où $b_{0}=(f, x)_{O}$. Alors il existe une factorisation $\partial f / \partial y=g_{1} \cdots g_{h}$ dans $\mathbb{C}\{x, y\}$ telle que :

(1) $\left(x, g_{k}\right)_{O}=b_{0} / e_{k}-b_{0} / e_{k-1}$ pour $k=1, \ldots, h$.

(2) Si $g$ est un facteur irréductible de $g_{k}$ alors

$$
\frac{(f, g)_{O}}{(x, g)_{O}}=\frac{e_{k-1} \bar{b}_{k}}{\bar{b}_{0}}
$$

Notons que la suite $\left(e_{k-1} \bar{b}_{k} / \bar{b}_{0}\right)$ où $k \in\{1, \ldots, h\}$ est strictement croissante et représente tous les invariants polaires de $f=0$ par rapport à $x=0$ (cf. [Me]). Ainsi dans le cas où $f$ est irréductible on a $Q(f, l)=$ $\left\{e_{k-1} \bar{b}_{k} / \bar{b}_{0}\right\}_{k=1}^{h}$ et de plus $g_{k}=j_{q}$ pour la valeur $k \in\{1, \ldots, h\}$ telle que $q=e_{k-1} \bar{b}_{k} / \bar{b}_{0}$. Cependant si $f$ est réduite non irréductible la décomposition 
de la courbe polaire $j(f, l)$ de [Ga] (qui est une généralisation du théorème ci-dessus) est plus fine que la décomposition $j(f, l)=\prod_{q \in Q(f, l)} j_{q}$ montrée dans l'introduction de cette note, comme le montre l'exemple donné à la page 17 de [Ga].

Dans la suite nous notons $\mathbb{C}\{x\}^{*}=\bigcup_{n>1} \mathbb{C}\left\{x^{1 / n}\right\}$ l'anneau des séries de Puiseux. Pour tout $y\left(x^{1 / n}\right)=\sum a_{i} x^{n_{i} / n} \in \mathbb{C}\left\{x^{1 / n}\right\}$ nous posons supp $y\left(x^{1 / n}\right)$ $=\left\{i: a_{i} \neq 0\right\}$, et pour $y\left(x^{1 / n}\right)=\sum a_{i} x^{n_{i} / n} \in \mathbb{C}\left\{x^{1 / n}\right\}-\{0\}$,

$$
\operatorname{ord} y\left(x^{1 / n}\right)=\frac{n_{i_{0}}}{n}, \quad \text { in } y\left(x^{1 / n}\right)=a_{i_{0}} x^{n_{i_{0}} / n}, \quad \text { inco } y\left(x^{1 / n}\right)=a_{i_{0}}
$$

où $i_{0}$ est le plus petit élement de $\operatorname{supp} y\left(x^{1 / n}\right)$.

La proposition ci-dessous se trouve déjà dans $[\mathrm{S}]$, [Ga, la première partie de la proposition 5.1] dans le cas où $g$ est une branche de la courbe polaire $\partial f / \partial y=0$ de $f(x, y)=0$.

Proposition 3.1. Fixons un $k \in\{1, \ldots, h\}$. Soit $g=g(x, y)$ une série irréductible, $y$-régulière d'ordre $p>0$. Supposons que $(x, g)_{0} \not \equiv 0\left(\bmod b_{0} / e_{k}\right)$ et $(f, g)_{0} /(x, g)_{0}>e_{k-2} \bar{b}_{k-1} / \bar{b}_{0}$ si $k>1$. Alors pour tout développement de Puiseux $z\left(x^{1 / p}\right) \in \mathbb{C}\left\{x^{1 / p}\right\}$ tel que $g\left(x, z\left(x^{1 / p}\right)\right)=0$ on a $b_{k} / b_{0} \notin \operatorname{supp} z\left(x^{1 / p}\right)$.

Démonstration. Considérons le cas $k=1$ et supposons que $(x, g)_{0} \not \equiv 0$ $\left(\bmod b_{0} / e_{1}\right)$. Montrons que $b_{1} / b_{0} \notin \operatorname{supp} z\left(x^{1 / p}\right)$. Sinon, il existe un entier $r>0$ tel que $b_{1} / b_{0}=r / p$ avec $p=(x, g)_{0}$, d'où $b_{1}(x, g)_{0} \equiv 0\left(\bmod b_{0}\right)$ et $\left(b_{1} / e_{1}\right)(x, g)_{0} \equiv 0\left(\bmod b_{0} / e_{1}\right)$. Puisque les entiers $b_{0} / e_{1}, b_{1} / e_{1}$ sont premiers entre eux nous avons $(x, g)_{0} \equiv 0\left(\bmod b_{0} / e_{1}\right)$, ce qui est une contradiction.

Passons maintenant au cas $k>1$. Par hypothèse $(f, g)_{0} /(x, g)_{0}>$ $e_{k-2} \bar{b}_{k-1} / \bar{b}_{0}$, alors $(x, g)_{0}=\left(b_{0} / e_{k-1}\right) q$ avec un entier $q>0$ (voir [GP, Lemma 5.1, p. 207]). Supposons que $b_{k} / b_{0} \in \operatorname{supp} z\left(x^{1 / p}\right)$. Alors nous pouvons écrire $b_{k} / b_{0}=r / p=r /(x, g)_{0}$ avec un entier $r>0$. Par conséquent $(x, g)_{0} b_{k}=r b_{0}$ et $\left(b_{0} / e_{k-1}\right) q b_{k}=r b_{0}$, d'où $q b_{k} \equiv 0\left(\bmod e_{k-1}\right)$ et $q b_{k} / e_{k} \equiv 0$ $\left(\bmod e_{k-1} / e_{k}\right)$. Les entiers $b_{k} / e_{k}, e_{k-1} / e_{k}$ sont premiers entre eux, d'où $q \equiv 0$ $\left(\bmod e_{k-1} / e_{k}\right)$. Donc $(x, g)_{0}=\left(b_{0} / e_{k-1}\right) q \equiv 0\left(\bmod b_{0} / e_{k}\right)$, ce qui est une contradiction.

Pour toute solution $y(t) \in \mathbb{C}\{t\}$ de l'équation $f\left(t^{n}, y\right)=0$ et pour tout $k \in\{1, \ldots, h\}$, notons $y_{k-1}(t)$ la somme de tous les termes de $y(t)$ de degré inférieur à $b_{k}$. Soit $f_{k-1}(x, y)=0$ l'équation minimale de $y_{k-1}(t)$; c'est un polynôme distingué irréductible tel que $f_{k-1}\left(t^{n}, y_{k-1}(t)\right)=0$ (voir [GP, p. 204]). Notons que $f\left(x, y\left(x^{1 / n}\right)\right)=0$ et $f_{k-1}\left(x, y_{k-1}\left(x^{1 / n}\right)\right)=0$.

LEMme 3.2. Écrivons $f(x, y)=u(x, y) \prod_{\varepsilon^{n}=1}\left(y-y\left(\varepsilon x^{1 / n}\right)\right)$ où $u(x, y)$ $\in \mathbb{C}\{x, y\}, u(0,0) \neq 0$. Soit $g(x, y) \in \mathbb{C}\{x, y\}$ une série irréductible, $y$-régulière et supposons que $z\left(x^{1 / p}\right) \in \mathbb{C}\left\{x^{1 / p}\right\}$ est un développement de Puiseux tel que $g\left(x, z\left(x^{1 / p}\right)\right)=0$. Soit $k \in\{1, \ldots, h\}$. Alors $(f, g)_{0} /(x, g)_{0}=$ $e_{k-1} \bar{b}_{k} / \bar{b}_{0}$ si et seulement si $\max \left\{\operatorname{ord}\left(z\left(x^{1 / p}\right)-y\left(\varepsilon x^{1 / n}\right)\right): \varepsilon^{n}=1\right\}=b_{k} / b_{0}$. 
Démonstration. Voir [S], [Z1], [Me] dans des coordonnées génériques et [GP, Lemma 3.4, p. 206] dans le cas général.

Proposition 3.3. Soit $g$ une série irréductible, y-régulière d'ordre $p>0$ telle que $(f, g)_{0} /(x, g)_{0}=e_{k-1} \bar{b}_{k} / \bar{b}_{0}$ pour $k>0$. Supposons que $(x, g)_{0} \not \equiv 0\left(\bmod b_{0} / e_{k}\right)$. Alors pour toute série de Puiseux $z\left(x^{1 / p}\right) \in$ $\mathbb{C}\left\{x^{1 / p}\right\}$ telle que $g\left(x, z\left(x^{1 / p}\right)\right)=0$ il existe $y\left(x^{1 / n}\right) \in \mathbb{C}\left\{x^{1 / n}\right\}$ telle que $f\left(x, y\left(x^{1 / n}\right)\right)=0$ et $\operatorname{ord}\left(z\left(x^{1 / p}\right)-y\left(x^{1 / n}\right)\right)=b_{k} / b_{0}$. De plus in $f\left(x, z\left(x^{1 / p}\right)\right)$ $=\operatorname{in} f\left(x, y_{k-1}\left(x^{1 / n}\right)\right)$.

Démonstration. Reprenons les notations du lemme 3.2. Soit $z\left(x^{1 / p}\right) \in$ $\mathbb{C}\left\{x^{1 / p}\right\}$ une série telle que $g\left(x, z\left(x^{1 / p}\right)\right)=0$. D'après la proposition 3.1 nous avons

$$
b_{k} / b_{0} \notin \operatorname{supp} z\left(x^{1 / p}\right) .
$$

De plus, la condition $(f, g)_{0} /(x, g)_{0}=e_{k-1} \bar{b}_{k} / \bar{b}_{0}$ entraîne, d'après le lemme 3.2 , l'existence d'une série $y\left(x^{1 / n}\right) \in \mathbb{C}\left\{x^{1 / n}\right\}$ telle que $f\left(x, y\left(x^{1 / n}\right)\right)=0$ et

$$
\operatorname{ord}\left(z\left(x^{1 / p}\right)-y\left(x^{1 / n}\right)\right)=b_{k} / b_{0} .
$$

D'après (1) et (2) le développement $z\left(x^{1 / p}\right)$ a les mêmes termes de degré plus petit que $b_{k} / b_{0}$ que la série $y\left(x^{1 / n}\right)$, et le terme $x^{b_{k} / b_{0}}$ n'apparaît pas dans $z\left(x^{1 / p}\right)$; nous pouvons donc écrire

$$
z\left(x^{1 / p}\right)=y_{k-1}\left(x^{1 / n}\right)+R_{k}\left(x^{1 / p}\right) \quad \text { où } \quad \text { ord } R_{k}\left(x^{1 / p}\right)>b_{k} / b_{0} .
$$

Par conséquent, nous obtenons

$$
z\left(x^{1 / p}\right)-y\left(\varepsilon x^{1 / n}\right)=\left(y_{k-1}\left(x^{1 / n}\right)-y\left(\varepsilon x^{1 / n}\right)\right)+R_{k}\left(x^{1 / p}\right)
$$

où ord $R_{k}\left(x^{1 / p}\right)>b_{k} / b_{0}$. Rappelons que $f_{k-1}\left(x, y_{k-1}\left(x^{1 / n}\right)\right)=0$ et $\left(f, f_{k-1}\right)_{0} /\left(x, f_{k-1}\right)_{0}=\bar{b}_{k} e_{k-1} / \bar{b}_{0}$ (voir [GP, Lemma 3.1, p. 204]); alors d'après le lemme 3.2 ,

$$
\max \left\{\operatorname{ord}\left(y_{k-1}\left(x^{1 / n}\right)-y\left(\varepsilon x^{1 / n}\right)\right): \varepsilon^{n}=1\right\}=b_{k} / b_{0} .
$$

Maintenant (4) et (5) impliquent

$$
\operatorname{in}\left(z\left(x^{1 / p}\right)-y\left(\varepsilon x^{1 / n}\right)\right)=\operatorname{in}\left(y_{k-1}\left(x^{1 / n}\right)-y\left(\varepsilon x^{1 / n}\right)\right)
$$

et nous obtenons

$$
\text { in } \begin{aligned}
f\left(x, z\left(x^{1 / p}\right)\right) & =u(0,0) \operatorname{in}\left(\prod_{\varepsilon^{n}=1}\left(z\left(x^{1 / p}\right)-y\left(\varepsilon x^{1 / n}\right)\right)\right) \\
& =u(0,0) \prod_{\varepsilon^{n}=1} \operatorname{in}\left(z\left(x^{1 / p}\right)-y\left(\varepsilon x^{1 / n}\right)\right) \\
& =u(0,0) \prod_{\varepsilon^{n}=1} \operatorname{in}\left(y_{k-1}\left(x^{1 / n}\right)-y\left(\varepsilon x^{1 / n}\right)\right) \\
& =\operatorname{in} f\left(x, y_{k-1}\left(x^{1 / n}\right)\right) .
\end{aligned}
$$


4. Démonstration du théorème principal. Nous allons utiliser deux ingrédients : le théorème de la factorisation de la polaire et la description de valeurs spéciales d'un pinceau.

Dans la suite nous supposons que $f=f(x, y) \in \mathbb{C}\{x, y\}$ est une série irréductible d'ordre plus grand que $1, y$-régulière de caractéristique $\left(b_{0}, \ldots, b_{h}\right)$.

Proposition 4.1. Fixons un $k \in\{1, \ldots, h\}$ et supposons que l'invariant polaire $e_{k-1} \bar{b}_{k} / \bar{b}_{0}=: N$ est un entier. Alors le pinceau $\left(f_{t}:=f-t x^{N}: t \in \mathbb{C}\right)$ a une seule valeur spéciale $t_{1} \neq 0$. De plus

$$
t_{1}=\left(\frac{f}{x^{N}}\right)\left(f_{k-1}\right)
$$

où $f_{k-1}(x, y)=0$ est une branche définie dans la section 3 .

Démonstration. D'après la proposition 2.2 toutes les valeurs spéciales non nulles du pinceau $\left(f_{t}:=f-t x^{N}: t \in \mathbb{C}\right)$ sont de la forme $\left(f / x^{N}\right)(g)$ où $g=0$ est une branche de la polaire $\partial f / \partial y=0$ telle que $(f, g)_{0} /(x, g)_{0}=$ $e_{k-1} \bar{b}_{k} / \bar{b}_{0}=N$. Dans la notation du théorème sur la factorisation de la polaire, $g$ divise $g_{k}$ et donc $(x, g)_{0} \leq\left(x, g_{k}\right)_{0}=b_{0} / e_{k}-b_{0} / e_{k-1}<b_{0} / e_{k}$, ce qui montre que $(x, g)_{0} \not \equiv 0\left(\bmod b_{0} / e_{k}\right)$.

Puisque $(f, g)_{0} /(x, g)_{0}=e_{k-1} \bar{b}_{k} / \bar{b}_{0}$, nous pouvons appliquer la proposition 3.3 aux séries $f$ et $g$. Soit $z\left(x^{1 / p}\right) \in \mathbb{C}\left\{x^{1 / p}\right\}$ une série de Puiseux telle que $g\left(x, z\left(x^{1 / p}\right)\right)=0$. On a donc inco $f\left(x, z\left(x^{1 / p}\right)\right)=\operatorname{inco} f\left(x, y_{k-1}\left(x^{1 / n}\right)\right)$. Il est facile à voir que $\left(f / x^{N}\right)(g)=$ inco $f\left(x, z\left(x^{1 / p}\right)\right)$ et

$$
\left(\frac{f}{x^{N}}\right)\left(f_{k-1}\right)=\operatorname{inco} f\left(x, y_{k-1}\left(x^{1 / n}\right)\right)
$$

ce qui démontre la proposition.

REMARQUE 4.2. La formule $t_{1}=\left(f / x^{N}\right)\left(f_{k-1}\right)$ montre que la valeur spéciale $t_{1}$ peut être calculée à l'aide des développements de Puiseux de la courbe irréductible $f(x, y)=0$. Plus précisément si $y\left(x^{1 / n}\right)=\cdots+$ $a_{b_{1}} x^{b_{1} / b_{0}}+\cdots+a_{b_{2}} x^{b_{2} / b_{0}}+\cdots+a_{b_{h}} x^{b_{h} / b_{0}}+\cdots$ alors

$$
t_{1}=\left(\frac{f}{x^{N}}\right)\left(f_{k-1}\right)=(-1)^{e_{k}} \frac{e_{0}}{e_{k-1}} u(0,0) a_{b_{1}}^{e_{0}-e_{1}} \cdots a_{b_{k-1}}^{e_{k-2}-e_{k-1}} a_{b_{k}}^{e_{k-1}} .
$$

Proposition 4.3. Soit $g=g(x, y) \in \mathbb{C}\{x, y\}$ une série irréductible, $y$ régulière telle que $(x, g)_{0}=(x, f)_{0}$. Supposons que $N:=(f, g)_{0} /(x, g)_{0} \in$ $Q(f, x)$ et $\eta(f, x)>N$. Alors $\eta(g, x)>N$.

Démonstration. On a $N=e_{k-1} \bar{b}_{k} / \bar{b}_{0}$ pour $k \neq h$. Soit $\left(b_{0}^{\prime}, \ldots, b_{h^{\prime}}^{\prime}\right)$ la caractéristique de $g$. Du lemme 3.2 et l'hypothèse $(x, g)_{0}=(x, f)_{0}$ il suit que $b_{0}^{\prime}=b_{0}, \ldots, b_{k-1}^{\prime}=b_{k-1}$ et $b_{k}^{\prime} \geq b_{k}$. Par conséquent $\overline{b_{k}^{\prime}} \geq \bar{b}_{k}$ et $\eta(g, x)=$ $e_{h^{\prime}-1}^{\prime} \overline{b_{h^{\prime}}^{\prime}} / \overline{b_{0}^{\prime}} \geq e_{k-1}^{\prime} \overline{b_{k}^{\prime}} / \overline{b_{0}^{\prime}} \geq e_{k-1} \bar{b}_{k} / \bar{b}_{0}=N$. 
L'égalité $\eta(g, x)=N$ entraînerait $\overline{b_{k}^{\prime}}=\bar{b}_{k}$ et $b_{k}^{\prime}=b_{k}$. La suite $\left(b_{0}^{\prime}, \ldots, b_{k}^{\prime}\right)$ $=\left(b_{0}, \ldots, b_{k}\right)$ serait la caractéristique de $g$, ce qui est impossible car $k<h$ et donc $\operatorname{pgcd}\left(b_{0}, \ldots, b_{k}\right)>1$.

Démonstration du théorème principal. Soit $\Phi$ une paire de séries telle que l'application $h \mapsto h \circ \Phi$ est un isomorphisme de l'anneau $\mathbb{C}\{x, y\}$. Alors $f_{t} \circ \Phi$ et $f_{t}$ ont les mêmes valeurs spéciales. Nous pouvons donc, remplaçant $\left(f_{t}: t \in \mathbb{C}\right)$ par $\left(f_{t-t_{0}}: t \in \mathbb{C}\right)$, supposer que $t_{0}=0$.

Considérons les quatre cas distingués dans l'énoncé:

(1) Si $\eta\left(f_{0}, l\right)<N$ alors le pinceau $\left(f_{t}: t \in \mathbb{C}\right)$ est équisingulier par la proposition 1.1(3).

(2) Si $\eta\left(f_{0}, l\right)=N$ alors 0 est une valeur générique en vertu de la proposition 1.1(2). Dans le cas où la courbe $f_{0}=0$ est singulière l'existence d'une seule valeur spéciale $t_{1} \neq 0$ est une conséquence de la proposition 4.1. Si la courbe $f_{0}=0$ est lisse alors $\eta\left(f_{0}, l\right)=1$ et la valeur $t_{1}$ est définie par la relation $d f_{0}=t_{1} d l(0)$.

(3) Si $\eta\left(f_{0}, l\right)>N$ et $N \notin Q\left(f_{0}, l\right)$ alors 0 est la seule valeur spéciale par la proposition 1.1(1), (2).

(4) Si $\eta\left(f_{0}, l\right)>N$ et $N \in Q\left(f_{0}, l\right)$ alors la branche $f_{0}=0$ n'est pas lisse. La valeur $t_{0}=0$ est spéciale par la proposition 1.1(2). L'existence d'une seule valeur spéciale $t_{1}$ non nulle est une conséquence de la proposition 4.1.

Pour montrer que toutes les fibres du pinceau $\left(f_{t}: t \in \mathbb{C} \backslash\left\{t_{0}, t_{1}\right\}\right)$ sont décomposables considérons $g=f_{t}$ avec un $t \notin\left\{t_{0}, t_{1}\right\}$. Il est facile à vérifier que $\left(f_{0}, g\right)_{0} /(x, g)_{0}=N$. D'autre part $\eta(g, x)=N$ d'après le corollaire 1.5. Si $g$ était irréductible alors on aurait $\eta(g, x)>N$ par la proposition 4.3, donc $g$ est décomposable.

5. Exemples. Voici des exemples qui illustrent le théorème principal:

(1) Soit $f(x, y)=0$ une branche de semi-groupe $\Gamma(f)=\left\langle\bar{b}_{0}, \ldots, \bar{b}_{h}\right\rangle$ où $\bar{b}_{0}=(f, l)_{0}$. Alors $\eta(f, l)=e_{h-1} \bar{b}_{h} / \bar{b}_{0}$. Considérons le pinceau d'Ephraim $f_{t}=f-t l^{\bar{b}_{0}}, t \in \mathbb{C}$. Ainsi le pinceau $\left(f_{t}: t \in \mathbb{C}\right)$ est équisingulier si et seulement si $e_{h-1} \bar{b}_{h}<\bar{b}_{0}^{2}$ (l'inégalité d'Abhyankar-Moh [AM] et [GP]). On a toujours $e_{h-1} \bar{b}_{h} \neq \bar{b}_{0}^{2}$. Si $e_{h-1} \bar{b}_{h}>\bar{b}_{0}^{2}$ alors le pinceau $\left(f_{t}: t \in \mathbb{C}\right)$ a une seule valeur spéciale $t_{0}=0$. Toutes les fibres $f_{t}$ avec $t \neq 0$ sont décomposables (voir [Eph, Corollary 2.2]). Ceci montre que pour un pinceau d'Ephraim, l'équisingularité du pinceau, l'inégalité de Abhyankar-Moh et l'irréductibilité de toutes les fibres (propriété de Moh, voir [Eph]) sont des propriétés équivalentes.

(2) Soit $f(x, y)=0$ la courbe irréductible de semi-groupe $\Gamma(f)=$ $\left\langle\bar{b}_{0}, \ldots, \bar{b}_{h}\right\rangle$. En particulier $\min Q(f, l)=\bar{b}_{1}$. Prenons le pinceau $f_{t}=f-$ $t l^{\bar{b}_{1}}$. Si $h=1$, d'après le théorème 1.8 le pinceau $\left(f_{t}\right)$ a une seule valeur 
spéciale $t_{1} \neq 0$. De plus la valeur de $\mu$ minimal pour le pinceau est $\mu^{\text {min }}=$ $\left(\bar{b}_{1}-1\right)\left(\bar{b}_{0}-1\right)$. Par contre si $h>1$ alors le pinceau a exactement deux valeurs spéciales : $t_{0}=0$ et $t_{1}$ non nulle.

(3) Irréductibilité des fibres génériques du pinceau. Dans les hypothèses du théorème 1.8 si $\eta\left(f_{t_{0}}, l\right)>N$ et $N \notin Q\left(f_{t_{0}}, l\right)$ alors $t_{0}$ est la seule valeur spéciale du pinceau $\left(f_{t}\right)$. Soit $f_{0}=y^{2}-x^{5}$ (donc $\eta\left(f_{0}, l\right)=5$ est le seul invariant polaire de $f_{0}$ ) et prenons le pinceau $f_{t}:=y^{2}-x^{5}-t x^{N}$. Si $N=2$ toutes les fibres $f_{t}$ associées aux valeurs non spéciales sont décomposables. Par contre si $N=3$ elles sont toutes irréductibles. Cet exemple nous permet dire que dans le cas (3) du théorème on ne peut pas dire comment sont les fibres génériques, car elles sont parfois décomposables (cet exemple pour $N=2$ ) et parfois irréductibles (le même exemple mais avec $N=3$ ).

(4) Branches dont tous les invariants polaires sont entiers.

(a) Pour tout entier $n \geq 0$ la suite $(6,8,11+6 n)$ est la caractéristique d'une branche plane $\gamma_{n}$. Le semi-groupe associé à $\gamma_{n}$ est $\langle 6,8,27+6 n\rangle$ et les invariants polaires sont $\{8,9+2 n\}$.

(b) La suite $(30,36,46,61)$ est la caractéristique d'une branche de semi-groupe $\Gamma=\langle 30,36,190,585\rangle$ et les invariants polaires sont $\{36,38,39\}$.

(5) Irréductibilité des fibres spéciales du pinceau. Dans les hypothèses du théorème $1.8 \mathrm{si} \eta\left(f_{t_{0}}, l\right)=N$ alors il existe une seule fibre spéciale du pinceau qui est différente de $f_{t_{0}}$. Soit $f_{0}=y^{3}+x^{4}+x^{5}$. Alors $f_{0}$ est irréductible et $Q(f, x)=\{4\}$. Considérons $f_{t}=f_{0}-t x^{4}$. Il y a une seule valeur spéciale $t_{1}=1$ et $f_{1}=y^{3}-x^{5}$ est irréductible. Cependant si nous prenons $f_{0}=$ $\left(y^{3}-x^{4}\right)^{2}-x^{9}$, alors $f_{0}$ est irréductible et $Q(f, x)=\{8,9\}$. Considérons $f_{t}=f_{0}-t x^{9}$. La seule valeur spéciale du pinceau est $t_{1}=-1$ et la fibre spéciale est $f_{-1}=\left(y^{3}-x^{4}\right)^{2}$, qui est non réduite. Cet exemple se généralise pour toute branche $f_{0}$ de caractéristique $\left(b_{0}, \ldots, b_{h}\right)$ avec $h>1$ telle que $x=0$ soit transverse à $f_{0}=0$, en utilisant la déformation de la courbe monomiale de $f_{0}=0$ (voir Appendice de Teissier dans [Z2]).

\section{Singularités à l'infini d'un polynôme de deux variables com-} plexes. Soit $F=F(x, y) \in \mathbb{C}[x, y]$ un polynôme de degré $N>1$ et notons $F^{*}=F^{*}(x, y, z) \in \mathbb{C}[x, y, z]$ la forme homogène correspondante à $F$. Notons $C$ la courbe projective (pas nécessairement réduite) d'équation $F^{*}(x, y, z)=0$ et $C_{\infty}$ l'ensemble des points à l'infini de $C$ défini par les équations $F^{*}(x, y, z)=0, z=0$. Pour décrire les singularités à l'infini de l'application polynomiale $F: \mathbb{C}^{2} \rightarrow \mathbb{C}$ considérons le pinceau

$$
C^{t}: F^{*}(x, y, z)-t z^{N}=0, \quad t \in \mathbb{C} .
$$

Pour tout point $O \in C_{\infty}$ le pinceau $C^{t}$ détermine le pinceau local 


$$
C_{O}^{t}: f_{O}-t l_{O}^{N}=0, \quad t \in \mathbb{C}
$$

où $f_{O}=0$ (resp. $l_{O}=0$ ) est une équation locale de $C$ (resp. de la droite à l'infini $\left.\mathbb{L}_{\infty}: z=0\right)$. Si la courbe $C$ est réduite nous notons $Q\left(C, \mathbb{L}_{\infty}\right)=$ $\bigcup_{O} Q_{O}\left(C, \mathbb{L}_{\infty}\right)$ où $Q_{O}\left(C, \mathbb{L}_{\infty}\right)$ est l'ensemble des invariants polaires de la courbe locale $C_{O}$ par rapport à la droite de l'infini au point $O \in C_{\infty}$.

Par convention $Q_{O}\left(C, \mathbb{L}_{\infty}\right)=\emptyset$ si $C$ et $\mathbb{L}_{\infty}$ s'intersectent en $O$ transversalement.

Finalement, notons $\Lambda_{O}(F)$ l'ensemble des valeurs spéciales du pinceau local $C_{O}^{t}, t \in \mathbb{C}$, et posons $\Lambda(F)=\bigcup_{O} \Lambda_{O}(F)$. Les éléments de $\Lambda(F)$ s'appellent valeurs critiques à l'infini de l'application polynomiale $F$ (voir [D]). Notons que si la courbe $C^{t}$ n'est pas réduite alors $t \in \Lambda(F)$. Si $\Lambda(F)=\emptyset$ on dit que le polynôme $F$ est bon.

La deuxième et la troisième partie de la proposition ci-dessous sont bien connues (voir [LVT], [NLVT], [CaP]).

Proposition 6.1. (1) Supposons que la courbe $C^{t_{0}}$ est réduite. Alors $\Lambda(F) \subset\left\{t_{0}\right\}$ si et seulement si $N \notin Q\left(C^{t_{0}}, \mathbb{L}_{\infty}\right)$.

(2) Pour tout $t_{0} \in \mathbb{C}: t_{0} \notin \Lambda(F)$ si et seulement si $C^{t_{0}}$ est réduite et $\sup Q\left(C^{t_{0}}, \mathbb{L}_{\infty}\right) \leq N($ par convention $\sup \emptyset=-\infty)$.

(3) Le polynôme $F$ est bon si et seulement si la courbe $C$ est réduite et $\sup Q\left(C, \mathbb{L}_{\infty}\right)<N$.

Démonstration. On applique la proposition 1.1 aux pinceaux locaux $C_{O}^{t}$, $t \in \mathbb{C}$, où $O \in C_{\infty}$.

Nous dirons que le polynôme $F \in \mathbb{C}[x, y]$ dépend d'une coordonnée (affine) si $F(x, y)=F^{0}(a x+b y+c)$ où $F^{0}$ est un polynôme d'une seule variable.

Nous omettons la démonstration simple du

Lemme 6.2. Supposons que le polynôme F n'a pas de facteurs multiples. Alors les conditions suivantes sont équivalentes :

(1) Le polynôme $F$ dépend d'une seule coordonnée.

(2) $C_{\infty}=\{O\}$ et $\left(C_{O}^{t}: t \in \mathbb{C}\right)$ est un pinceau de droites passant par le point $O$.

(3) $C_{\infty}=\{O\}$ et $\mu_{O}(C)=(N-1)^{2}$.

Nous pouvons maintenant démontrer la

Proposition 6.3. Supposons que $C_{\infty}=\{O\}$ et que $C$ est réduite. Alors $\min Q_{O}\left(C, \mathbb{L}_{\infty}\right) \leq N$. Les conditions suivantes sont équivalentes:

(1) $\min Q_{O}\left(C, \mathbb{L}_{\infty}\right)=N$.

(2) Le polynôme $F$ est sans facteurs multiples et dépend d'une seule coordonnée.

(3) $Q_{O}\left(C, \mathbb{L}_{\infty}\right)=\{N\}$. 
Démonstration. Fixons un entier $N>0$. Il suffit de montrer que la condition $N \leq \min Q_{O}\left(C, \mathbb{L}_{\infty}\right)$ implique que le polynôme $F$ dépend d'une seule coordonnée.

En effet, si $N \leq \min Q_{O}\left(C, \mathbb{L}_{\infty}\right)$ alors en utilisant la remarque 2.3 nous trouvons que $\mu^{\min }=(N-1)^{2}$. Soit $t_{0}$ une valeur régulière. D'après le lemme 6.2 le polynôme $F-t_{0}$ dépend d'une seule coordonnée; alors il en est de même pour $F$.

Finalement, on peut dire que si $C$ est une courbe réduite avec un seul point à l'infini $O \in \mathbb{L}_{\infty}$ alors le pinceau $\left(C_{O}^{t}: t \in \mathbb{C}\right)$ est dégénéré au sens $[\mathrm{MM}]$ si et seulement si c'est un pinceau de droites passant par $O$.

EXEMPLES. Les exemples ci-dessous sont donnés par différents auteurs mais sans calcul des invariants polaires à l'infini.

(1) Soit $F(x, y)=y^{2 k}(x+y)^{2}+(x+y) y, k>1$ (voir [CaP, p. 47]). Les points à l'infini sont $O_{1}=(1: 0: 0)$ et $O_{2}=(1:-1: 0)$. On a $Q_{O_{1}}\left(C, \mathbb{L}_{\infty}\right)=\left\{\frac{4 k^{2}}{2 k-1}\right\}$ et $Q_{O_{2}}\left(C, \mathbb{L}_{\infty}\right)=\{4 k\}$. Donc $\Lambda(F)=\Lambda_{O_{2}}(F)=\{0\}$ car $\operatorname{deg} F=2 k+2$.

(2) Soit $F(x, y)=x^{2} y+x y^{2}+x^{5} y^{3}+x^{3} y^{5}$ (voir [D, p. 15]). Il y a quatre points à l'infini mais c'est seulement en $O_{1}=(0: 1: 0)$ et $O_{2}=(1: 0: 0)$ que la multiplicité d'intersection de $C$ avec $\mathbb{L}_{\infty}$ est plus grande que 1 . On a $Q_{O_{1}}\left(C, \mathbb{L}_{\infty}\right)=Q_{O_{2}}\left(C, \mathbb{L}_{\infty}\right)=\{15 / 2\}$ et le polynôme $F$ est bon car $\operatorname{deg} F=8$.

(3) Soit $F(x, y)=y^{2 n}+x^{3 n} y^{n}(x+y)^{n}+x^{4} y$ (voir [LVTO, p. 418]). Il y a trois points à l'infini $O_{1}=(0: 1: 0), O_{2}=(1: 0: 0)$ et $O_{3}=(1:-1: 0)$. On a $Q_{O_{1}}\left(C, \mathbb{L}_{\infty}\right)=\{3 n\}, Q_{O_{2}}\left(C, \mathbb{L}_{\infty}\right)=\{5 n\}$ et $Q_{O_{3}}\left(C, \mathbb{L}_{\infty}\right)=\{3 n\}$. Alors $\Lambda_{O_{1}}\left(C, \mathbb{L}_{\infty}\right)=\Lambda_{O_{3}}\left(C, \mathbb{L}_{\infty}\right)=\emptyset$. La valeur $t_{0}=0$ est regulière mais $\Lambda_{O_{2}}\left(C, \mathbb{L}_{\infty}\right) \neq \emptyset$. De plus $\sharp \Lambda_{O_{2}}\left(C, \mathbb{L}_{\infty}\right) \leq\left(C, \mathbb{L}_{\infty}\right)_{0}-1=n-1$ d'après le corollaire 1.7. On vérifie directement que $\Lambda_{O_{2}}\left(C, \mathbb{L}_{\infty}\right)=n-1$, donc $\sharp \Lambda(F)=n-1$.

Remerciements. Nous sommes reconnaissants à Bernard Teissier pour les discussions fructueuses sur le sujet et pour l'intérêt montré pour ce travail.

Pendant la préparation de ce travail, le deuxième auteur a bénéficié du soutien de l'Université de La Laguna et de la DGUI du gouvernement des Iles Canaries, et de l'hospitalité du Departamento de Matemática Fundamental de La Laguna.

\section{Références}

[AM] S. S. Abhyankar and T. Moh, Embeddings of the line in the plane, J. Reine Angew. Math. 276 (1975), 148-166. 
[Ca] E. Casas Alvero, Singularities of Plane Curves, London Math. Soc. Lecture Note Ser. 276, Cambridge Univ. Press, 2000.

[CaP] E. Casas Alvero and R. Peraire, A bound for the number of critical values at infinity, J. Pure Appl. Algebra 142 (1999), 35-47.

[D] A. H. Durfee, Five definitions of critical point at infinity, dans : Singularities: the Brieskorn Anniversary Volume, Progr. Math. 162, Birkhäuser, 1998, $345-360$.

[Eph] R. Ephraim, Special polars and curves with one place at infinity, dans : Proc. Sympos. Pure Math. 40, Part I, Amer. Math. Soc., 1983, 353-359.

[Ga] E. García Barroso, Sur les courbes polaires d'une courbe plane réduite, Proc. London Math. Soc. 81 (2000), 1-28.

[GP] J. Gwoździewicz and A. Płoski, On the approximate roots of polynomials, Ann. Polon. Math. 3 (1995), 199-210.

[LMW] D. T. Lê, F. Michel et C. Weber, Courbes polaires et topologie des courbes planes, Ann. Sci. École Norm. Sup. 24 (1991), 141-169.

[LR] D. T. Lê and C. P. Ramanujam, The invariance of Milnor's number implies the invariance of the topological type, Amer. J. Math. 98 (1973), 67-78.

[LW] D. T. Lê et C. Weber, Équisingularité dans les pinceaux de germes de courbes planes et $C^{0}$-suffisance, Enseign. Math. 43 (1997), 355-380.

[LVT] Lê Van Thành, Affine polar quotients and singularity at infinity of an algebraic plane curve, dans : Singularity Theory (Trieste, 1991), D. T. Lê et al. (eds.), World Sci., 1995, 336-344.

[LVTO] Lê Van Thành and M. Oka, Note on estimation of the number of the critical values at infinity, Kodai Math. J. 17 (1994), 409-419.

$[\mathrm{MM}] \quad$ H. Maugendre et F. Michel, Fibrations associées à un pinceau de courbes planes, Ann. Fac. Sci. Toulouse Math. 10 (2001), 745-777.

[Me] M. Merle, Invariants polaires des courbes planes, Invent. Math. 41 (1977), $103-111$.

[NLVT] W. D. Neumann and Lê Van Thành, On irregular links at infinity of algebraic planes curves, Math. Ann. 295 (1993), 239-244.

[S] H. J. S. Smith, On the higher singularities of plane curves, Proc. London Math. Soc. 6 (1875), 153-182.

[T1] B. Teissier, Varietés polaires. I. Invariants polaires des singularités des hypersurfaces, Invent. Math. 40 (1977), 267-292.

[T2] -, Cycles évanescents, sections planes et condition de Whitney, Astérisque 7-8 (1973), 285-362.

[T3] - Polyèdre de Newton Jacobien et équisingularité, dans : Séminaire sur les Singularités, Publ. Math. Univ. Paris VII 7, 1980, 193-221.

[Z1] O. Zariski, General theory of saturation and of saturated local rings II. Saturated local rings of dimension 1, Amer. J. Math. 93 (1971), 872-964.

[Z2] - Le problème des modules pour les branches planes, Hermann, Paris, 1986.

Departamento de Matemática Fundamental

Facultad de Matemáticas

Universidad de La Laguna

38271 La Laguna, Tenerife, España

E-mail: ergarcia@ull.es
Department of Mathematics Technical University Al. 1000 L PP 7 25-314 Kielce, Poland E-mail: matap@tu.kielce.pl 